\title{
Atomic structure of a thin silica film on a Mo(112) substrate: A combined experimental and theoretical study
}

\author{
T. K. Todorova, ${ }^{1}$ M. Sierka, ${ }^{1} *$ J. Sauer, ${ }^{1}$ S. Kaya, ${ }^{2}$ J. Weissenrieder, ${ }^{2}$ J.-L. Lu, ${ }^{2,3}$ H.-J. Gao, ${ }^{3}$ S. Shaikhutdinov, ${ }^{2}$ and \\ H.-J. Freund ${ }^{2}$ \\ ${ }^{1}$ Humboldt-Universität zu Berlin, Institut für Chemie, Unter den Linden 6, 10099 Berlin, Germany \\ ${ }^{2}$ Fritz-Haber-Institut der Max-Planck-Gesellschaft, Faradayweg 4-6, 14195 Berlin, Germany \\ ${ }^{3}$ Institute of Physics, Chinese Academy of Sciences P. O. Box 603, Beijing 100080, China \\ (Received 4 November 2005; revised manuscript received 12 January 2006; published 21 April 2006)
}

\begin{abstract}
The atomic structure of the thin $\mathrm{SiO}_{2}$ film on a Mo(112) substrate has been determined based on a combination of density functional theory calculations and high-quality experimental data obtained from scanning tunneling microscopy, infrared reflection absorption spectroscopy, and x-ray photoelectron spectroscopy. The film consists of a honeycomblike, two-dimensional network of corner-sharing $\left[\mathrm{SiO}_{4}\right]$ tetrahedra. One oxygen atom of each tetrahedron binds to the Mo(112) substrate and is located in a bridge position between Mo atoms located in rows protruding from the metal surface. The other three oxygen atoms form Si-O-Si bonds with the neighboring tetrahedra.
\end{abstract}

DOI: 10.1103/PhysRevB.73.165414

PACS number(s): 68.55.-a, 68.60.-p, 81.15.-z, 68.90.+g

\section{INTRODUCTION}

Thin oxide films grown on a metal substrate attract increasing interest due to their applications as insulating layers in integrated circuits, protective films against corrosion, or as supports for metal nanoparticles in sensors and catalysts. Such films can be grown with a thickness varying from a few angstroms to as much as $100 \AA$ (Refs. 1-3 and references therein). One of the increasingly important applications of the thin films is their use as model supports for studying structure-reactivity relationships on supported oxides and oxide supported metal particles. Their advantage is that all surface science techniques relying on good electrical and thermal conductivity of the samples can be applied without any restrictions (e.g., charging problems). In order to gain control over the structural aspects of the model catalysts, it is advantageous to use thin crystalline films instead of amorphous ones. However, only in very few cases are crystalline films with a known atomic structure obtained. ${ }^{4,5}$

One of the most important oxides in advanced technology is silica $\left(\mathrm{SiO}_{2}\right)$. It is widely used as a support material in heterogeneous catalysis, but the preparation of clean and well-ordered crystalline films on a metal surface was reported only recently by Schroeder et al. ${ }^{6,7}$ Modifying the original recipe, Goodman et al. ${ }^{8-12}$ have successfully grown highly crystalline thin $\mathrm{SiO}_{2}$ films on a $\mathrm{Mo}(112)$ substrate. However, the detailed atomic structure of this film remained unknown. Based on density functional theory (DFT) calculations, Ricci and Pacchioni have proposed a $\beta$-cristobalite derived structure with a thickness of three atomic layers as the most stable model for the film. ${ }^{13}$ Using analogies of vibrational spectra of the film to known compounds, Goodman and co-workers have suggested that the film consists of a layer of isolated $\left[\mathrm{SiO}_{4}\right]$ clusters arranged in a $c(2 \times 2)$ structure on the Mo(112) surface with all oxygen atoms bonding to the Mo substrate. ${ }^{9,12}$ However, none of these models successfully explained all known experimental data.

Here, we use DFT to determine the atomic structure of the ultrathin, crystalline $\mathrm{SiO}_{2}$ film on a $\mathrm{Mo}(112)$ substrate and demonstrate that this structure is compatible with the results of a multitude of surface science techniques: scanning tunneling microscopy (STM), photoelectron spectroscopy (XPS), and infrared reflection absorption spectroscopy (IRAS). The structure consists of a two-dimensional (2D) honeycomblike network of $\mathrm{SiO}_{4}$ tetrahedra, with one oxygen of each tetrahedron binding to the protruding Mo atoms of the metal surface, while the other three form Si-O-Si bonds with the neighboring tetrahedra. The topmost surface layer made up of inert Si-O-Si bonds explains the essential inertness of the film, in particular towards water. ${ }^{14}$ We demonstrate an excellent agreement between calculated and highquality experimental data for this form of silica, which resembles the sheet structure of monolayer silicates. ${ }^{15}$

In a recent communication, ${ }^{16}$ we briefly reported the main aspects of our findings. Independently, a similar structure model of the film was proposed by Giordano et al. ${ }^{17}$ Here, we present detailed computational and experimental results that led to the successful determination of this unknown film structure. We also demonstrate discrepancies between calculated and experimental data for previously proposed models and add results on antiphase domain boundaries. The paper is organized as follows. In Sec. II we briefly describe the experimental setup used to prepare and characterize the $\mathrm{SiO}_{2} / \mathrm{Mo}(112)$ system. The computational methods applied are presented in Sec. III A followed by a short description of the substrate model (Sec. III B) and the formalism used to compare the stability of the investigated structures (Sec. III C). Discussion of the experimental and theoretical results is given in Secs. IV and V, respectively.

\section{EXPERIMENTAL METHODS}

The experiments were carried out in an ultrahigh vacuum (UHV) chamber (base pressure below $1 \times 10^{-10} \mathrm{mbar}$ ), equipped with a low-energy electron diffractometer (LEED, Omicron), x-ray photoelectron spectrometer (XPS, Scienta SES-200), IR spectrometer (Bruker IFS $66 \mathrm{v} / \mathrm{s}$ ), scanning 
tunneling microscope (STM, Omicron), and standard facilities for surface cleaning. Sample heating was performed by electron bombardment from a thoriated tungsten filament placed close to the backside of the sample. The temperature was measured by a tungsten-rhenium (W-5\%Re/W-26\%Re) thermocouple spot-welded to the edge of the Mo crystal. The Mo(112) (99.99\%, Mateck) single crystal was cleaned via cycles of annealing in $1 \times 10^{-6} \mathrm{mbar}_{2}$ at $800 \mathrm{~K}$ followed by a flash to $2300 \mathrm{~K}$ in UHV, until XPS and LEED measurements indicated a clean, well-ordered surface. Silicon was evaporated onto the clean Mo substrate from a Si rod, $2 \mathrm{~mm}$ in diameter, using a commercial electron-beam assisted evaporator (Focus EFM3) at a deposition rate of $\sim 0.07$ monolayer (ML) per minute as measured by XPS. During evaporation, the sample was kept on the same potential as the $\mathrm{Si}$ rod in order to prevent $\mathrm{Si}$ ions from being accelerated towards the surface. The films were prepared by exposing the clean $\mathrm{Mo}(112)$ surface to $5 \times 10^{-8}$ mbar of $\mathrm{O}_{2}(99.9995 \%$, Linde) at $900 \mathrm{~K}$ for $5 \mathrm{~min}$ and subsequent deposition of approximately $1.2 \mathrm{ML}$ of $\mathrm{Si}$ at the same temperature and oxygen pressure. The films were then annealed at elevated temperatures, up to $1250 \mathrm{~K}$, in UHV for $5 \mathrm{~min}$. The infrared reflection absorption spectra (IRAS) were measured with $p$-polarized light at $84^{\circ}$ grazing angle of incidence (resolution $\sim 2 \mathrm{~cm}^{-1}$ ). The binding energies (BE) in the XPS spectra were calibrated relative to the Fermi edge of the clean Mo crystal. The spectra were measured at normal electron emission. STM images were taken at room temperature and the bias voltage is given relative to the sample. The images were subjected to plane correction and low-pass filtering.

\section{COMPUTATIONAL DETAILS}

\section{A. Methods}

All calculations are based on density functional theory (DFT) and performed using the Vienna Ab-Initio Simulation Package (VASP). ${ }^{18,19}$ We employed a plane-wave basis set with an energy cutoff of $400 \mathrm{eV}$ along with the PerdewWang (PW91) (Ref. 20) exchange-correlation functional. The electron-ion interactions were described by the projector augmented wave (PAW) method ${ }^{21}$ in the implementation of Kresse and Joubert. ${ }^{22}$ Unless stated otherwise, the integrations of the first Brillouin zone used an $(8 \times 4 \times 1)$ Monkhorst-Pack grid. ${ }^{23}$ The positions of nuclei were relaxed until the forces were smaller than $1 \times 10^{-3} \mathrm{eV} / \AA$.

The core-level energies were calculated including final state effects using a modified projector augmented wave method. The STM images were simulated from the selfconsistent charge density employing the Tersoff-Hamann approach. ${ }^{24}$ Calculations of the vibrational spectra within the harmonic approximation used a central finite difference method with $0.02 \AA$ displacements of the atoms in each Cartesian direction. The intensities were obtained from the derivatives of the dipole moment component perpendicular to the surface.

It is known that the agreement between calculated harmonic vibrational frequencies and observed fundamentals can be improved using an empirical scaling factor derived from calculated and observed frequencies of known compounds. ${ }^{25}$ We have chosen $\alpha$-quartz as the reference substance and performed full optimization of lattice constants and atomic positions using the same setup, except for a higher plane-wave energy cutoff of $800 \mathrm{eV}$. For the final configuration, the relaxation of atomic positions and calculation of harmonic frequencies were performed using a $400 \mathrm{eV}$ cutoff. The experimental phonon frequencies of $\alpha$-quartz in the range $400-1200 \mathrm{~cm}^{-1}$ were taken from Refs. 26 and 27. Longitudinal optical (LO) modes are not considered because they depend on the longitudinal macroscopic field that is not included in the calculations. ${ }^{28}$ Using a least square fitting procedure, we have derived the scaling factor of 1.0312, with the root mean square error (rms) of $13.6 \mathrm{~cm}^{-1}$ between calculated and observed frequencies (see Supporting Information in Ref. 16).

\section{B. Substrate Model}

The model of $\mathrm{Mo}(112)$ substrate is constructed from relaxed bulk bcc Mo structure with calculated lattice constant of a=3.155 $\AA$, in excellent agreement with the experimental value of $3.147 \AA$. The surface unit cell is modeled using an orthorhombic $(2 \times 2)$ supercell with the lattice constants $a_{0}$ $=5.464 \AA$ and $b_{0}=8.923 \AA$. The slab contains seven Mo layers; three bottom layers are fixed at their bulk positions and remaining four are relaxed.

\section{Stability of the Models}

The stability of different $\mathrm{SiO}_{2} / \mathrm{Mo}(112)$ film models is compared using the formation energy, $\Delta E_{\text {form }}$, from a clean Mo(112) surface, bulk $\alpha$-quartz, and oxygen,

$$
\mathrm{Mo}(112)+m\left(\mathrm{SiO}_{2}\right)_{\alpha-\text { quartz }}+n \frac{1}{2} \mathrm{O}_{2} \rightarrow\left(\mathrm{SiO}_{2}\right)_{m} n \mathrm{O} / \mathrm{Mo}(112),
$$

where $m$ and $n$ are the number of $\mathrm{SiO}_{2}$ units and oxygen excess in a surface unit cell, respectively. Thus, $\Delta E_{\text {form }}$ is defined as

$$
\begin{aligned}
\Delta E_{\text {form }}= & E^{\left(\mathrm{SiO}_{2}\right)_{m} n \mathrm{O} / \mathrm{Mo}(112)} \\
& -\left(E^{\mathrm{Mo}(112)}+m E^{\left(\mathrm{SiO}_{2}\right)_{\alpha-\text { quartz }}}+n \frac{1}{2} E^{\mathrm{O}_{2}}\right),
\end{aligned}
$$

where $E^{\left(\mathrm{SiO}_{2}\right)_{m} n \mathrm{O} / \mathrm{Mo}(112)}$ corresponds to the energy of a given

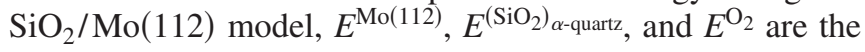
energies of the clean Mo(112) surface, bulk $\alpha$-quartz, and an oxygen molecule, respectively. Since different models differ by their chemical composition, the relative stability has to be compared at a given $\mathrm{O}_{2}$ partial pressure corresponding to experimental conditions. We use the free energy change of reaction (1) per surface area $S, \Delta \gamma$,

$$
\Delta \gamma(T, p)=\frac{1}{S}\left[\Delta E_{\text {form }}-n \Delta \mu_{\mathrm{O}}(T, p)\right],
$$

with $\Delta \mu_{\mathrm{O}}(T, p)=\mu_{\mathrm{O}}-1 / 2 E_{\mathrm{O}_{2}}$, where $\mu_{\mathrm{O}}$ is the oxygen chemical potential. The free energy is calculated neglecting zero-point energy, vibrational entropy, and enthalpy contri- 




FIG. 1. (Color online) (a) STM image of a well-ordered silica film showing the absence of holes and silica particles on the film. The LEED pattern (negative) of the silica film is shown as inset. Line defects propagating in the [110] direction of $\mathrm{Mo}(112)$ are indicated by arrows in (b). High-resolution STM images are shown in (c) and (d), which depend on the tunneling parameters. Image size and tunneling parameters are: (a) $75 \times 75 \mathrm{~nm}^{2}, V_{\mathrm{s}}=2 \mathrm{~V}, I$ $=0.2 \mathrm{nA}$; (b) $14 \times 14 \mathrm{~nm}^{2}, V_{\mathrm{s}}=1.3 \mathrm{~V}, I=0.45 \mathrm{nA}$; (c) $5 \times 2.5 \mathrm{~nm}^{2}$, $V_{\mathrm{s}}=0.65 \mathrm{~V}, I=0.8 \mathrm{nA}$; (d) $5 \times 2.5 \mathrm{~nm}^{2}, V_{\mathrm{s}}=1.2 \mathrm{~V}, I=0.35 \mathrm{nA}$.

butions. This approximation has been shown to be sufficiently accurate for metal oxides. ${ }^{29}$ The oxygen chemical potential can be related to temperature and partial oxygen pressure by assuming that the surface is in thermodynamic equilibrium with the gas phase $\mathrm{O}_{2} \cdot{ }^{29}$

\section{EXPERIMENTAL RESULTS}

LEED studies of the silica films showed that film ordering strongly depends on the annealing temperature (see also Ref. 6) such that high-temperature annealing at $\sim 1250 \mathrm{~K}$ results in a diffraction pattern with very sharp spots showing a $c(2$ $\times 2)-M o(112)$ structure [see inset in Fig. 1(a)]. STM images of the annealed films revealed smooth terraces of $20-50 \mathrm{~nm}$ in width and step height of $1.2 \AA$ or multiple thereof, which corresponds to monatomic steps of the Mo substrate underneath the film. These films are found to be free of holes and silica particles. In addition, STM images revealed antiphase domain boundaries propagating in the $[\overline{1} 10]$ direction as shown in Fig. 1(b).

The high-resolution STM images show a honeycomblike structure of the silica film with a periodicity of $\sim 5.2 \AA$ in the [311] direction and $\sim 5.5 \AA$ in the [1 $1 \overline{1} 1]$ direction, in agreement with the $c(2 \times 2)$ structure observed by LEED. However, it has turned out that the atomic structure seen in STM depends on the tunneling conditions [cf. Figs. 1(c) and 1(d)]. This indicates that different electronic states can be involved in the tunneling process, which requires theoretical calculations of the electronic structure and simulations of these STM images (see Sec. V D below).

Figure 2(a) shows a series of IRAS spectra as a function of annealing temperature. The as-deposited, nonannealed
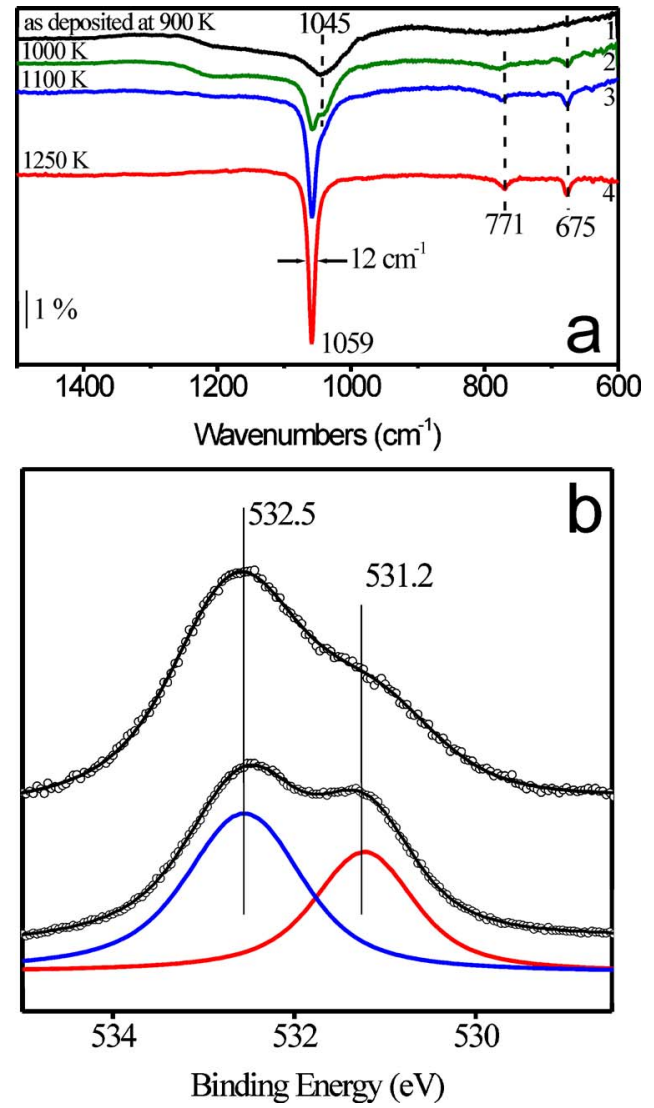

FIG. 2. (Color online) (a) IRAS spectra of silica films as a function of annealing temperature as indicated. The well-annealed film is characterized by a very sharp peak at $1059 \mathrm{~cm}^{-1}$ and small peaks at 771 and $675 \mathrm{~cm}^{-1}$. (b) The O $1 \mathrm{~s}$ region of XPS spectra obtained for the film as deposited at $900 \mathrm{~K}$ and for the film annealed at $1250 \mathrm{~K}$. The last spectrum is deconvoluted into two components with BEs centered at 532.5 and $531.2 \mathrm{eV}$ and with an integral ratio of $3: 2$.

films exhibit a main absorption band centered at $\sim 1045 \mathrm{~cm}^{-1}$ with a broad feature extended up to $1250 \mathrm{~cm}^{-1}$. These bands gradually attenuate upon annealing, while the peaks at 1059, 771, and $675 \mathrm{~cm}^{-1}$ gain intensity. The main signal at $1059 \mathrm{~cm}^{-1}$ becomes very sharp such that the full width half maximum (FWHM) is only $12 \mathrm{~cm}^{-1}$, suggesting a high ordering of the film and a single phonon excitation. Curves 2 and 3 in Fig. 2(a) are basically formed by superposition of spectra 1 and 4, with a corresponding weighing factor, which implies coexistence of two structures on the surface at intermediate annealing temperature.

The XPS study of these films showed only a single peak in the Si $2 p$ region with a binding energy (BE) of $103.2 \mathrm{eV}$ (not shown here), which is characteristic for the $\mathrm{Si}^{4+}$ oxidation state. ${ }^{30}$ However, the $\mathrm{O} 1 s$ region revealed two different chemical environments for the oxygen ions [Fig. 2(b)], with $\mathrm{BEs}$ at 532.5 and $531.2 \mathrm{eV}$. Both values are significantly higher than those observed for $\mathrm{MoO}_{x}$ films formed on the Mo(112) surfaces [ $\sim 530 \mathrm{eV}$ (Ref. 31)], and therefore cannot be assigned to the oxidation of the Mo substrate underneath the film. Annealing in UHV at $1250 \mathrm{~K}$ reduces the Si $2 p$ intensity simultaneously with the signal of oxygen at 

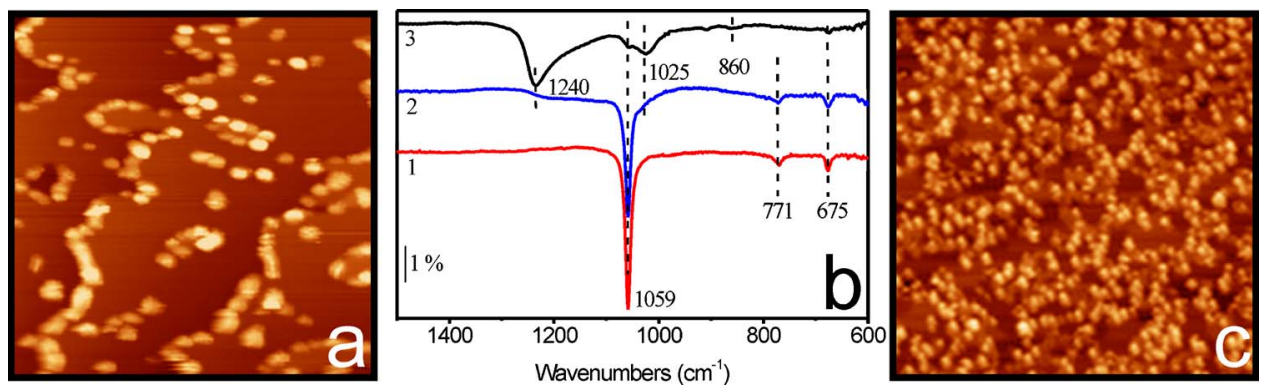

FIG. 3. (Color online) STM images and IRAS spectra of silica particles formed on the well-ordered silica film by Si deposition in 5 $\times 10^{-8}$ mbar $\mathrm{O}_{2}$ at $900 \mathrm{~K}$. Spectra 2 and 3 correspond to the STM images (a) and (c), respectively. Spectrum 1 is for the original well-ordered film (see Fig. 2). Image size and tunneling parameters: (a) $100 \times 100 \mathrm{~nm}^{2}, V_{\mathrm{S}}=2.8 \mathrm{~V}$ and $I=0.2 \mathrm{nA}$; (c) $100 \times 100 \mathrm{~nm}{ }^{2}, V_{\mathrm{S}}$ $=3.5 \mathrm{~V}$ and $I=0.1 \mathrm{nA}$.

$532.5 \mathrm{eV}$, which can be explained by the desorption of silica clusters at elevated temperatures. The intensity ratio of the two $\mathrm{O}$ species at higher and lower BEs in well-ordered films is found to be $3: 2$. Spectra taken in the Mo $3 d$ region show no sign of oxidation of the supporting Mo surface. However, if the last annealing step to $1250 \mathrm{~K}$ is performed under $10^{-7}$ mbar $\mathrm{O}_{2}$ pressure it produces a partially oxidized Mo surface as evidenced by components on the high BE side of the metallic component in Mo $3 d$.

In order to link spectral features and a surface morphology, we have performed a combined STM and IRAS study as follows. We have first prepared a well-ordered silica film by annealing at $1250 \mathrm{~K}$. Then Si was deposited onto this film at $900 \mathrm{~K}$ in oxygen ambient, which resulted in the formation of silica clusters decorating steps and line defects of the film as shown in Fig. 3(a). This led to attenuation of the main peak at $1059 \mathrm{~cm}^{-1}$ and emerging of a broad band between 1250 and $1000 \mathrm{~cm}^{-1}$ [spectrum 2 in Fig. 3(b)]. When larger amounts of $\mathrm{Si}$ were deposited, particles were observed to cover most parts of the terraces [Fig. 3(c)]. As a result, the IRAS peaks belonging to the original silica film are almost vanished. Instead, two relatively broad bands centered at 1240 and $1025 \mathrm{~cm}^{-1}$ are observed [spectrum 3 in Fig. 3(b)]. Annealing of these samples to $1250 \mathrm{~K}$ basically restores both the morphology and IRAS spectrum of the well-ordered film.

These experiments clearly show that IRAS features beyond those at 1059,771 , and $675 \mathrm{~cm}^{-1}$ originate from threedimensional silica particles. The band at $1240 \mathrm{~cm}^{-1}$, observed for the high density of silica particles, is within the range of asymmetric $\mathrm{Si}-\mathrm{O}$ stretching frequencies reported for bulk silica, whereas the features at around $860 \mathrm{~cm}^{-1}$ are their symmetric counterparts. ${ }^{32}$ Concomitantly, the peak at $1025 \mathrm{~cm}^{-1}$, which appears as a shoulder at low particle density and is more pronounced at high particle coverage, can be associated with silica clusters located at line defects such as step edges and antiphase domain boundaries. Turning back to Fig. 2, the dependence of the IRAS spectra on annealing temperature can be rationalized on the basis of silica wetting the metal substrate and simultaneous desorption of the more weakly bound silica particles at elevated temperatures.

\section{THEORETICAL RESULTS AND DISCUSSION}

\section{A. Construction of structure models}

In selecting possible structure models for the $\mathrm{SiO}_{2} / \mathrm{Mo}(112)$ film we used criteria based on the known experimental evidences: (i) periodicity consistent with the LEED and STM data; (ii) hexagonal arrangement of the building units suggested by the STM results; (iii) simple atomic structure or/and high symmetry of the film suggested by XPS and IRAS data; (iv) fully saturated surface, as indicated by chemical inertness of the film towards $\mathrm{H}_{2} \mathrm{O}, \mathrm{O}_{2}$, and $\mathrm{CO}$ (Ref. 14); and (v) an average thickness of the film of about 5-8 $\AA$ as determined by angular resolved XPS. ${ }^{6}$ Inspired by the structures of layered silicates, ${ }^{15}$ we have constructed models consisting of double (2 ML) and single (1 ML) monolayers of $\left[\mathrm{SiO}_{4}\right]$ tetrahedra. None of the considered models shows dangling $\mathrm{Si}-\mathrm{O}$ bonds or edge-shared $\mathrm{SiO}_{4}$ tetrahedra strained at the film surface. Two of the $2 \mathrm{ML}$ models have a precise $\mathrm{SiO}_{2}$ stoichiometry. Additionally, we considered thirteen $2 \mathrm{ML}$ models with oxygen excess and following surface unit cell compositions: $\left(\mathrm{SiO}_{2}\right)_{8} \mathrm{O}$, $\left(\mathrm{SiO}_{2}\right)_{8} 2 \mathrm{O}$, and $\left(\mathrm{SiO}_{2}\right)_{8} 3 \mathrm{O}$. Figure 4 shows two examples of the most stable $2 \mathrm{ML}$ models considered. The $1 \mathrm{ML}$ models are formally constructed by cutting off the bottom $\mathrm{Si}$ and $\mathrm{O}$ atomic layers of $2 \mathrm{ML}$ stoichiometric film in such a way that
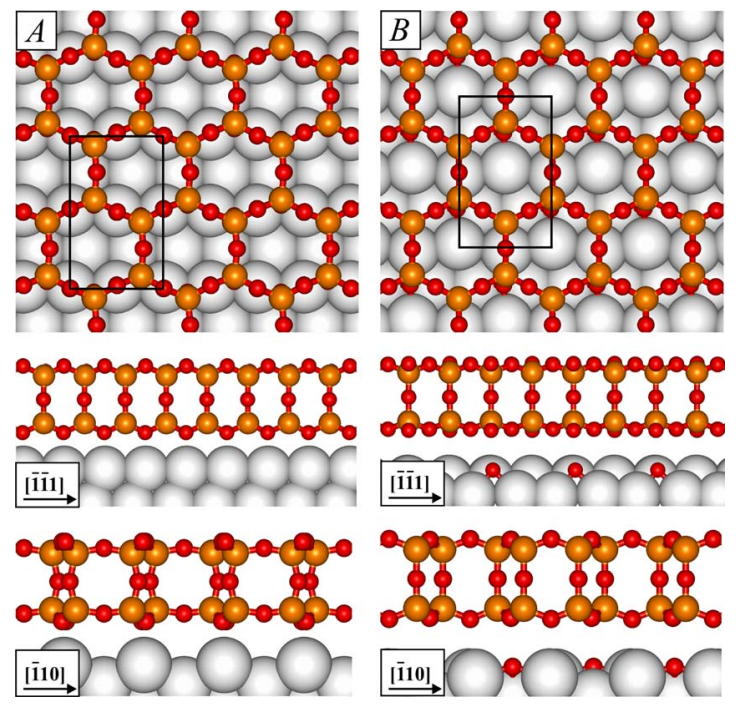

FIG. 4. (Color online) The $2 \mathrm{ML} \mathrm{SiO}_{2} / \mathrm{Mo}$ (112) film models: (a) stoichiometric $\left(\mathrm{SiO}_{2}\right)_{8} / \mathrm{Mo}(112)$ model with $\mathrm{O}$ atoms in top position on Mo atoms along [1]11] direction, and (b) $\left(\mathrm{SiO}_{2}\right)_{8} 2 \mathrm{O} / \mathrm{Mo}(112)$ model with excess $\mathrm{O}$ atoms in bridge sites located in the trenches. 

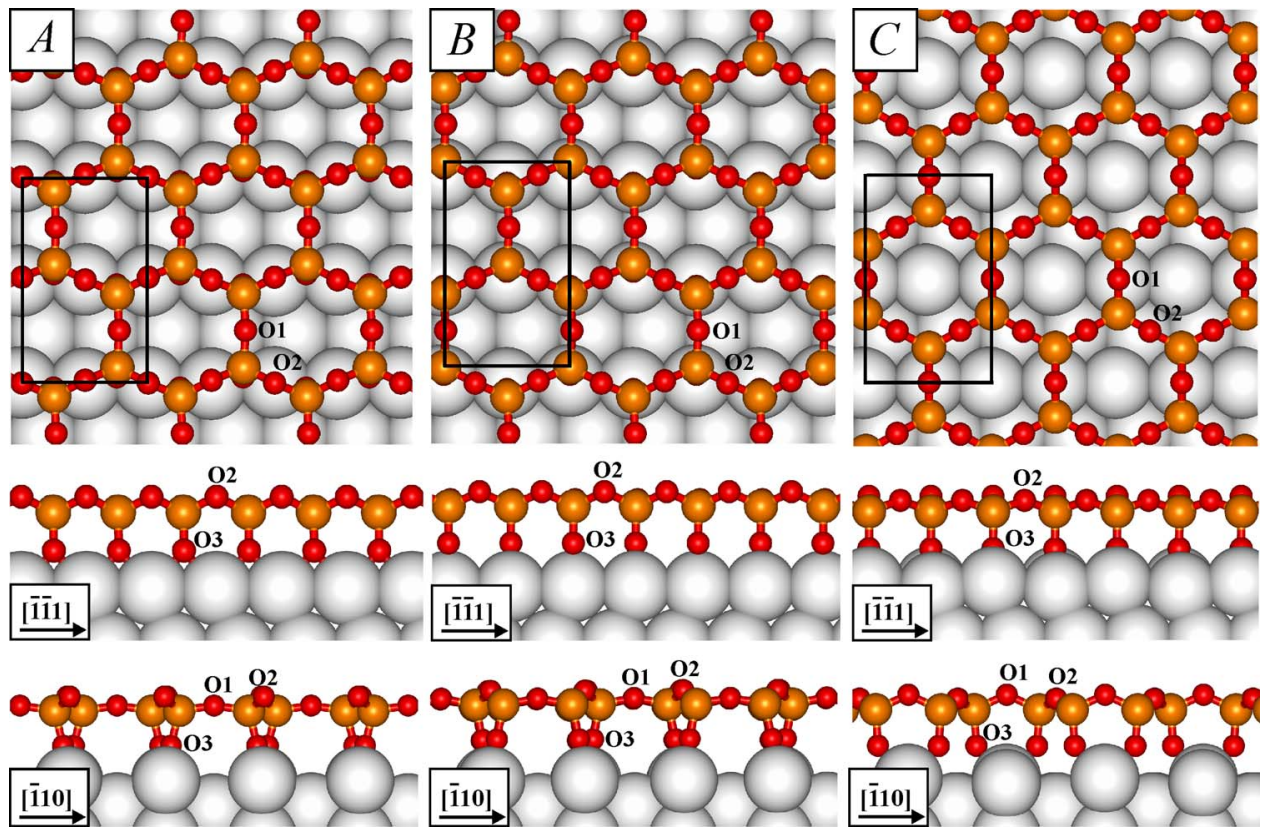

oxygen atoms are pointing to the metal substrate (see Fig. 5). For this film, three possible orientations with respect to the Mo(112) surface are possible: $(A)$ oxygen atoms in bridge sites, $(B)$ top sites, and $(C)$ pseudo threefold hollow sites with respect to the rows of protruding Mo atoms running along the $[\overline{1} 11]$ direction. It should be mentioned that these structures show some similarities with the proposed, but rejected, model 4 of Ref. 9 . We also considered the recently proposed models of isolated $c(2 \times 2)$-[ $\left.\mathrm{SiO}_{4}\right]$ tetrahedra with surface unit cell composition $\left(\mathrm{SiO}_{2}\right)_{2} 4 \mathrm{O}$ [Fig. 6(a)] (Ref. 9) and the $\beta$-cristobalite film with the composition $\left(\mathrm{SiO}_{2}\right)_{10} 3 \mathrm{O} .{ }^{13}$

\section{B. Structure}

Figure 4 shows the two most stable structures of the $2 \mathrm{ML}$ silica film models. The stoichiometric $\left(\mathrm{SiO}_{2}\right)_{8}$ film has oxygen atoms from the $\mathrm{Si}-\mathrm{O}-\mathrm{Si}$ chains along [1111] direction at top position with respect to the Mo protruding atoms [Fig. 4(a)]. As a result of full valence saturation of the bottom and top sides of the silica layer, this film stays unbound to the substrate and is separated by $2.4 \AA$ from the topmost Mo atoms of the Mo(112) surface. Attempts to bind the film to the substrate by introducing oxygen excess resulted in unbound silica film and oxidized Mo(112) surface. Figure 4(b) shows the most stable example of such a film, with the surface unit cell composition $\left(\mathrm{SiO}_{2}\right)_{8} 2 \mathrm{O}$ and the excess oxygen atoms located in bridge positions in trenches along the [110] direction.

Table I presents selected calculated structural parameters for the three $1 \mathrm{ML} \mathrm{SiO}_{2} / \mathrm{Mo}(112)$ models (see Fig. 5 for atom labeling). The formal surface unit cell composition of all three models is $\left(\mathrm{SiO}_{2}\right)_{4} 2 \mathrm{O} / \mathrm{Mo}(112)$, which suggests nonstoichiometric films. However, one oxygen atom of each $\mathrm{SiO}_{4}$ tetrahedron forms an O-Mo bond and formally shares one electron with the metal surface. Thus, the structure of the
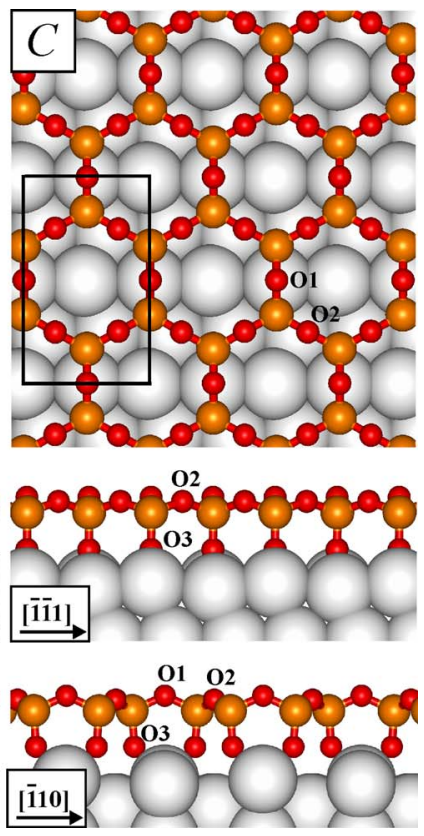

FIG. 5. (Color online) The three $1 \mathrm{ML}$ models of the $\mathrm{SiO}_{2} / \mathrm{Mo}(112)$ film. Black rectangle indicates the $\left(\mathrm{SiO}_{2}\right)_{4} 2 \mathrm{O}$ surface unit cell. film can also be interpreted as a stoichiometric layer of molybdenum silicate deposited on the Mo(112) surface. The distance between the plane of the topmost $\mathrm{O} 2$ atoms and the plane between the topmost Mo atoms of the Mo(112) rows and the Mo atoms in the furrows is $4.5 \AA$. In all the models,


FIG. 6. (Color online) (a) Isolated $c(2 \times 2)-\left[\mathrm{SiO}_{4}\right]$ tetrahedra structure of Chen et al. Black rectangle indicates the $\left(\mathrm{SiO}_{2}\right)_{2} 4 \mathrm{O}$ surface unit cell; (b) simulated STM image with $V_{\mathrm{s}}=0.65 \mathrm{~V}$; (c) simulated IRAS spectrum and the IR active vibrational normal modes. $\mathrm{O}$ and $\mathrm{Si}$ atoms are represented as red and orange spheres, respectively. The Mo(112) surface is drawn as black sticks. 
TABLE I. Selected structural parameters $\left(\AA\right.$, deg) of the 1 ML models of the $\mathrm{SiO}_{2} / \mathrm{Mo}(112)$ film (see Fig. 5 for atom labeling).

\begin{tabular}{lccccccc}
\hline \hline Model & Si-O1 & Si-O2 & Si-O3 & Mo-O3 & Si-O1-Si & Si-O2-Si & Si-O-Mo \\
\hline 1 ML $A$ & 1.62 & 1.64 & 1.65 & 2.11 & 163.2 & 132.9 & $139.2,139.6$ \\
1 ML $B$ & 1.62 & 1.64 & 1.64 & 1.91 & 157.1 & 134.4 & 177.3 \\
1 ML $C$ & 1.63 & 1.63 & 1.65 & $2.22,2.30,2.32$ & 131.5 & 145.3 & $121.9,121.1,152.1$ \\
\hline \hline
\end{tabular}

the $\mathrm{Si}-\mathrm{O}$ bond lengths show only a small variation in the range of $1.62-1.65 \AA$. On the other hand, the bonds between surface Mo and interface oxygen (O3) atoms differ significantly among the three models. They range from $1.91 \AA$ for the $1 \mathrm{ML}(\operatorname{model} B)$ to $2.32 \AA$ for $1 \mathrm{ML}(\operatorname{model} C)$. The $\mathrm{Si}-\mathrm{O} 1-\mathrm{Si}$ bond angle shows much larger variation $\left(131.5-163.2^{\circ}\right)$ than the $\mathrm{Si}-\mathrm{O} 2-\mathrm{Si}$ angle $\left(132.9-145.3^{\circ}\right)$. The Si-O-Mo bond angle varies in a range of $121.1-177.3^{\circ}$. Note, that the $1 \mathrm{ML}$ model $B$ with top positions of interface $\mathrm{O}$ atoms with respect to the protruding Mo rows corresponds to the model independently found by Giordano et al. ${ }^{17}$

Figure 6(a) displays the structure suggested by Chen et al. ${ }^{9}$ It consists of isolated $\mathrm{SiO}_{4}$ tetrahedra located in the trenches of the $\operatorname{Mo}(112)$ substrate with all four oxygen atoms bound to the metal surface. Two of the oxygen atoms bind to Mo atoms of the protruding rows and the other two are located in the trenches. The $\mathrm{Si}$ atoms from every $\mathrm{SiO}_{4}$ unit are positioned downwards with respect to the topmost pairs of $\mathrm{O}$ atoms [see Fig. 6(c)]. The $\mathrm{Si}-\mathrm{O}$ bond lengths are in the range of $1.65-1.73 \AA$, slightly longer than the bonds in the $1 \mathrm{ML}$ $\mathrm{SiO}_{2} / \mathrm{Mo}(112)$ models, whereas the bonds to the Mo atoms (2.14-2.28 $\AA$ ) are similar. The Si-O-Mo bond angles that involve Mo atoms from the protruding rows are much smaller $\left(75.3-107.7^{\circ}\right)$ than the corresponding ones in the monolayer films and increase to $119.6-162.4^{\circ}$ for the angles involving Mo atoms from the trenches.

\section{Stability}

Figure 4(a) shows the most stable stoichiometric $2 \mathrm{ML}$ model. It has a positive value of the formation energy $\left[\Delta E_{\text {form }}=0.8 \mathrm{eV} ; m=8, n=0\right.$, cf. Eq. (1)]. The partially oxidized Mo(112) surface [Fig. 4(b), $m=8, n=2$ ] results in a quite stable $2 \mathrm{ML}$ structure with the formation energy of $\Delta E_{\text {form }}=-6.5 \mathrm{eV}$ but unbound film. The increase in the stability of the $2 \mathrm{ML}$ model with increasing oxygen content is certainly connected with high oxidation energy of Mo. Among the $1 \mathrm{ML}$ models, the highest stability shows structure $A\left(\Delta E_{\text {form }}=-10.8 \mathrm{eV} ; m=4, n=2\right)$ which is $2 \mathrm{eV}$ more stable than models $B\left(\Delta E_{\text {form }}=-8.8 \mathrm{eV}\right)$ and $C\left(\Delta E_{\text {form }}=\right.$ $-8.7 \mathrm{eV})$. The $\beta$-cristobalite (three-layer) film structure proposed by Ricci and Pacchioni is $1.7 \mathrm{eV}$ less stable $\left(\Delta E_{\text {form }}\right.$ $=-9.1 \mathrm{eV} ; m=10, n=3)$ than monolayer model $A$, whereas the model of isolated $c(2 \times 2)-\left[\mathrm{SiO}_{4}\right]$ clusters proposed by Chen et al. is slightly more stable $\left(\Delta E_{\text {form }}=-11.5 \mathrm{eV} ; m=2\right.$, $n=4)$.

In order to determine the most stable structure at the experimental conditions, we have plotted the surface-related free energy of formation $\Delta \gamma$ [see Eq. (3)] for the calculated models as a function of oxygen chemical potential $\Delta \mu_{\mathrm{O}}(T, p)$ at $T=300$ and $1200 \mathrm{~K}$ (Fig. 7). Our calculations suggest that $1 \mathrm{ML}$ model $A$ is thermodynamically the most favorable structure at all experimentally relevant oxygen pressures. The free energy of formation of the second most stable $1 \mathrm{ML}$ model $B$ is by $43 \mathrm{meV} / \AA^{2}$ larger and differs only by $0.6 \mathrm{meV} / \AA^{2}$ from the $1 \mathrm{ML} C$ film. Since these models have the same excess of oxygen content $n$ [See Eq. (1)], the three lines on the plot are parallel. The isolated $c(2 \times 2)-\left[\mathrm{SiO}_{4}\right]$ tetrahedra model is energetically more favorable than the $\beta$-cristobalite $\mathrm{SiO}_{2}$ film on $\mathrm{Mo}(112)$ for oxygen chemical potential values higher than about $-2.5 \mathrm{eV}$. However, when compared to the $1 \mathrm{ML}$ model $A$ it becomes more stable only at very high oxygen partial pressures (e.g., $\sim 3 \times 10^{8}$ atm at $T=1200 \mathrm{~K})$. Thus, based on the calculated free energy changes, we conclude that the $1 \mathrm{ML} A$ structure is the most probable candidate for the atomic model of the $\mathrm{SiO}_{2} / \mathrm{Mo}(112)$ film.

\section{Properties}

In order to verify whether the model of the 2D network of $\mathrm{SiO}_{4}$ tetrahedra indeed agrees with the experimental findings,

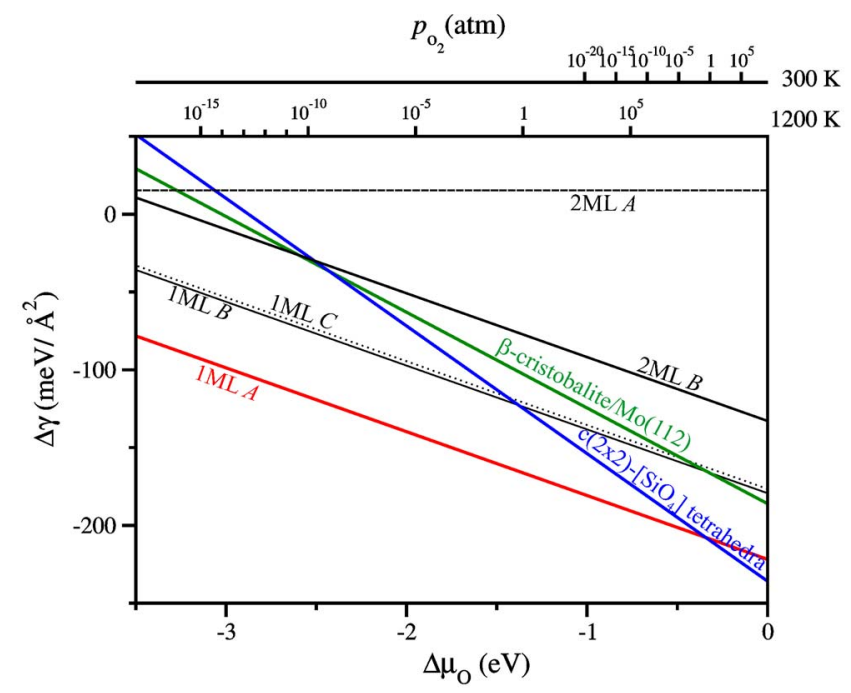

FIG. 7. (Color online) Surface-related free energy of formation $\Delta \gamma(T, p)$ for the most stable models of the $\mathrm{SiO}_{2} / \mathrm{Mo}(112)$ film as a function of oxygen chemical potential $\Delta \mu_{\mathrm{O}}: 2 \mathrm{ML}$ and $1 \mathrm{ML}$ models, $\beta$-cristobalite film (Ref. 13), and the isolated $c(2 \times 2)-\left[\mathrm{SiO}_{4}\right]$ tetrahedra model (Ref. 9). In the top $x$ axis, the dependence on $\Delta \mu_{\mathrm{O}}(T, p)$ has been cast into a pressure scale at fixed temperatures of $T=300$ and $1200 \mathrm{~K}$. 
TABLE II. Calculated scaled [scaling factor 1.0312 (see text for details)] harmonic frequencies $\left(\mathrm{cm}^{-1}\right)$ and IR intensities (in parentheses, normalized to 1.0) of the most intense vibrational modes for the three $\mathrm{SiO}_{2} / \mathrm{Mo}(112) 1 \mathrm{ML}$ films. Experimental IRAS frequencies are given for comparison.

\begin{tabular}{|c|c|c|c|c|c|c|c|}
\hline \multicolumn{2}{|c|}{$1 \mathrm{ML} A$} & \multicolumn{2}{|c|}{$1 \mathrm{ML} B$} & \multicolumn{2}{|c|}{$1 \mathrm{ML} C$} & \multicolumn{2}{|c|}{ Experiment } \\
\hline $\mathrm{Si}^{16} \mathrm{O}_{2}$ & $\mathrm{Si}^{18} \mathrm{O}_{2}$ & $\mathrm{Si}^{16} \mathrm{O}_{2}$ & $\mathrm{Si}^{18} \mathrm{O}_{2}$ & $\mathrm{Si}^{16} \mathrm{O}_{2}$ & $\mathrm{Si}^{18} \mathrm{O}_{2}$ & $\mathrm{Si}^{16} \mathrm{O}_{2}$ & $\mathrm{Si}^{18} \mathrm{O}_{2}$ \\
\hline $1061(0.91)$ & 1021 & $1132(1.00)$ & 1085 & $978(0.58)$ & 948 & 1059 & 1018 \\
\hline $779(0.07)$ & 770 & $781(0.04)$ & 774 & $804(0.004)$ & 795 & 771 & 764 \\
\hline $672(0.09)$ & 650 & 709 (0.13) & 689 & $646(0.09)$ & 616 & 675 & 656 \\
\hline $312(0.02)$ & 307 & $335(0.02)$ & 326 & $290(0.006)$ & 284 & & \\
\hline
\end{tabular}

we have calculated IR spectra and O $1 s$ core-level shifts as well as simulated the STM images at different tunneling conditions for all three $1 \mathrm{ML}$ structures $A, B$, and $C$. In addition, some properties of the monolayer model of isolated $c(2$ $\times 2)-\left[\mathrm{SiO}_{4}\right]$ clusters $^{9}$ are discussed.

Figure 2 shows the experimental IRAS spectra. The wellannealed silica film is characterized by a very sharp peak at $1059 \mathrm{~cm}^{-1}$ and small peaks at 771 and $675 \mathrm{~cm}^{-1}$. Table II shows calculated scaled harmonic frequencies of the most intense vibrational modes for the $1 \mathrm{ML}$ film models. The longitudinal macroscopic field is not included and, therefore, only transverse (TO) modes are obtained. For all three $1 \mathrm{ML}$ structures $A, B$, and $C$ there are only three IR modes above $400 \mathrm{~cm}^{-1}$ with non-negligible intensity. While for models $B$ and $C$ the calculated frequencies deviate by up to $75 \mathrm{~cm}^{-1}$ from the experimental values, for the $1 \mathrm{ML} A$ model the positions and relative intensities of the calculated bands are in excellent agreement with the experimental results [Fig. 2(a)]. Moreover, our calculations for the 1 ML $A$ model reproduce even the experimental ${ }^{18} \mathrm{O}$ isotopic shifts (Table II). As the observed IRAS spectrum of the film can be reproduced with calculated TO frequencies only, we conclude that LO modes do not contribute to the film spectrum.

The normal modes of the three most intense vibrations are visualized in Fig. 8. The mode at $1061 \mathrm{~cm}^{-1}$ is the in-phase combination of asymmetric Si-O-Mo stretching modes originating from the $\mathrm{Si}-\mathrm{O}$ bond pointing downwards to the Mo substrate. The mode at $779 \mathrm{~cm}^{-1}$ is the $\mathrm{Si}-\mathrm{O}-\mathrm{Si}$ symmetric stretching coupled with $\mathrm{Si}-\mathrm{O}-\mathrm{Si}$ bending, and the third mode at $672 \mathrm{~cm}^{-1}$ is a coupling of Si-O-Si bending with a small contribution of the Si-O-Si symmetric stretching modes. The vibration with a low IRAS intensity at $312 \mathrm{~cm}^{-1}$ corresponds to a vertical motion of the entire silica film. For the $1 \mathrm{ML}$ model $A$, the calculated IRAS inactive vibrations include combinations of Si-O-Si asymmetric stretching modes in the range of 1008 to $1195 \mathrm{~cm}^{-1}$, out-of-phase combinations of Si-O-Mo asymmetric stretching vibrations between 863 and
$912 \mathrm{~cm}^{-1}$ as well as coupled $\mathrm{Si}-\mathrm{O}-\mathrm{Si}$ symmetric stretching and Si-O-Si bending modes between 676 and $821 \mathrm{~cm}^{-1}$. Vibrations between 325 and $557 \mathrm{~cm}^{-1}$ include complicated couplings of bending modes of the film. Generally, the assignment of vibrational modes below $900 \mathrm{~cm}^{-1}$ is difficult due to a strong coupling between stretching and different bending modes.

The vibrational spectrum calculated for the film of isolated $c(2 \times 2)-\left[\mathrm{SiO}_{4}\right]$ clusters [Figure 6(c)] shows two almost equally intense peaks at 706 (coupled symmetric O-Si-O stretching and $\mathrm{O}-\mathrm{Si}-\mathrm{O}$ bending mode) and $532 \mathrm{~cm}^{-1}$ (coupled Mo-O stretching and $\mathrm{O}-\mathrm{Si}-\mathrm{O}$ bending) as well as a less intense peak at $403 \mathrm{~cm}^{-1}$ (Mo-O stretching and $\mathrm{O}-\mathrm{Si}-\mathrm{O}$ bending), which clearly disagree with the experimental spectra. The O-Si-O asymmetric stretching modes at 929 and $939 \mathrm{~cm}^{-1}$ involving topmost oxygen atoms bound to the protruding $\mathrm{Mo}$ rows as well as O-Si-O asymmetric stretching modes at 760 and $762 \mathrm{~cm}^{-1}$ involving oxygen atoms located in the furrows of the Mo(112) surface are IRAS inactive. This is because the $\mathrm{Si}-\mathrm{O}$ bonds in this model are aligned almost parallel to the surface and their stretching does not produce any change of the dipole moment perpendicular to the surface. Recently, Yakovkin reported calculation of the vibrational spectrum for the same model of isolated $c(2 \times 2)-\left[\mathrm{SiO}_{4}\right]$ tetrahedra, but using only a small molecular model of the surface. ${ }^{33}$ The Hartree-Fock method applied by the author along with a small STO-3G basis set is known to be particularly inaccurate for description of transition metals containing compounds. The Si-O stretching frequency reported at $1044 \mathrm{~cm}^{-1}$ corresponds to IRAS inactive modes at 929 and $939 \mathrm{~cm}^{-1}$ in our slab calculation.

Furthermore, we have calculated the difference in BEs of O $1 s$ core levels between different oxygen atoms present in the $1 \mathrm{ML}$ film models. For the $\mathrm{O} 1$ and $\mathrm{O} 2$ atoms in Si-OSi bridging positions [see Figs. 5(a)-5(c)], the BEs are nearly equal $( \pm 0.1 \mathrm{eV})$. The $\mathrm{BE}$ difference between $\mathrm{O} 1(\mathrm{O} 2)$ and $\mathrm{O} 3$ atoms is much larger and found to be $1.3,1.9$, and $1.1 \mathrm{eV}$ for



FIG. 8. (Color online) Vibrational normal modes viewed along [ $[\overline{1} 1]$ direction for the $1 \mathrm{ML}$ model $A$. Calculated and experimental (in parentheses) vibrational frequencies $\left(\mathrm{cm}^{-1}\right)$ are shown for comparison. The $\mathrm{O}$ and $\mathrm{Si}$ are represented by red and orange spheres, respectively. For clarity, the $\operatorname{Mo}(112)$ surface is drawn as sticks. 


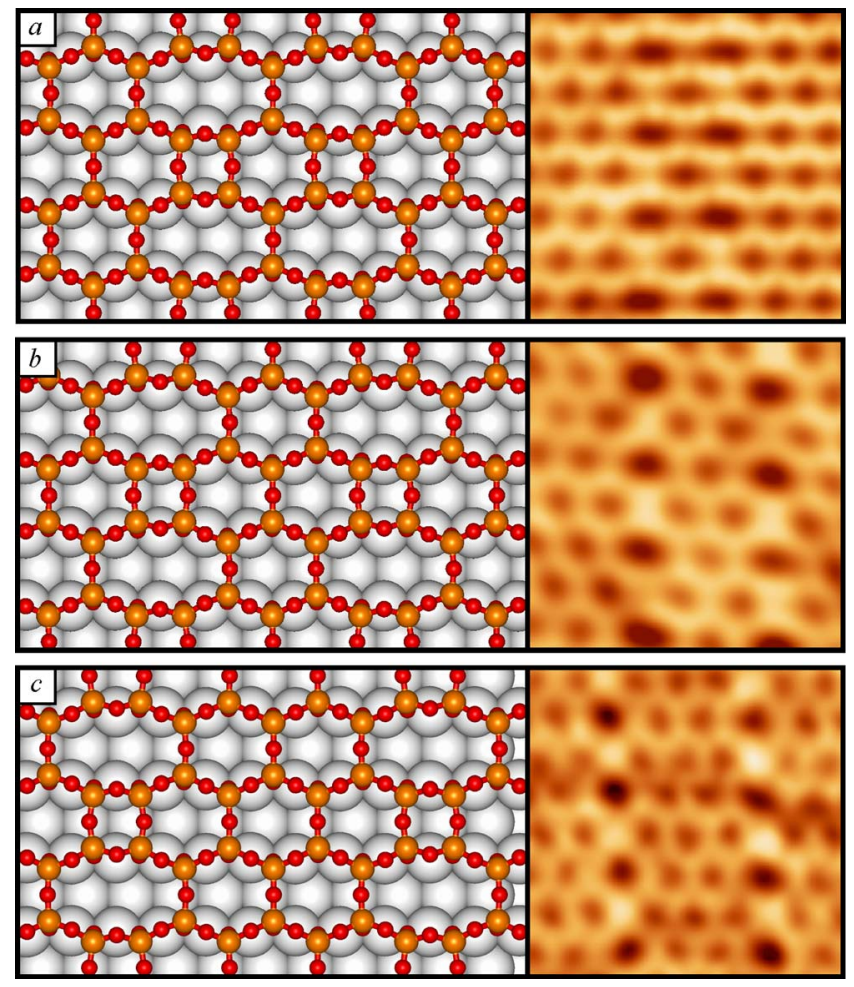

FIG. 9. (Color online) Simulated models of ADB (left) and experimental STM images (right, $3.5 \times 3.5 \mathrm{~nm}^{2}$ ): (a) sharing edge; tunneling parameters: $V_{\mathrm{s}}=1.2 \mathrm{~V}, I=0.4 \mathrm{nA}$; (b) separated by one six-membered ring; $V_{\mathrm{s}}=2.78 \mathrm{~V}, I=0.42 \mathrm{nA}$; and (c) separated by two six-membered rings; $V_{\mathrm{s}}=3.0 \mathrm{~V}, I=0.6 \mathrm{nA}$.

the models $A, B$ and $C$, respectively. The experimentally observed value for the $\mathrm{O} 1 s$ binding energy shift $(1.3 \mathrm{eV})$ again favors the monolayer model $A$. In contrast, the largest $\mathrm{BE}$ difference between the four unequivalent oxygen atoms of the $c(2 \times 2)-\left[\mathrm{SiO}_{4}\right]$ isolated cluster model is $0.8 \mathrm{eV}$.

Finally, based on calculated local density of states, we have simulated STM images of the most stable 1 ML $A$ structure at tunneling conditions applied in the experiments. The insets in Figs. 1(c) and 1(d) show the atomically resolved STM images (left) and simulated images (right) when the tunneling gap is set to $6 \AA$ at $1.2 \mathrm{~V}$ in Fig. 1(c) and $4 \AA$ at $0.65 \mathrm{~V}$ in Fig. 1(d). Interestingly, the protrusions imaged by STM are attributed to the $\mathrm{Si}$ atoms at a large tip-surface distance and to the bridging oxygen atoms at a smaller distance. In both cases, the simulated images match very well the experimental ones, thus providing further strong evidence for the $1 \mathrm{ML}$ model $A$. The simulated STM image of the isolated $c(2 \times 2)-\left[\mathrm{SiO}_{4}\right]$ clusters shows no dependence on the tip-surface separation. The image differs substantially from our experimental data, showing basically the whole $\mathrm{SiO}_{4}$ clusters as bright protrusions [see Figs. 6(a) and 6(b)].

\section{E. Structure and stability of antiphase domain boundaries}

As mentioned in Sec. IV, the experimental atomically resolved STM images reveal surface dislocations in a form of antiphase domain boundaries (ADB). Here, as a final evidence we demonstrate that our $1 \mathrm{ML}$ model $A$ of the $\mathrm{SiO}_{2} / \mathrm{Mo}(112)$ film provides a straightforward explanation of the structure of ADB.
The model of ADB can be constructed in the following way. The 1 ML $A$ film is cut along the $[\overline{1} 10]$ direction. Then, one-half of the structure is translated by half of the unit cell in the [ $\overline{1} \overline{1} 1]$ direction towards the other half. This results in formation of a chain of interchanging octahedral and square rings containing 8 and $4 \mathrm{Si}$ atoms, respectively.

In order to evaluate the stability of our ADB model, three supercells built from the $1 \mathrm{ML}$ model $A$ are constructed (cf. Fig. 9). We use unit cells with various sizes in order to simulate different separations between the antiphase domain boundaries. We start with an $(8 \times 2)$ unit cell that has two octahedrons sharing an edge in the [1̄11] direction [Fig. 9(a)]. In the $(5 \times 2)$ and $(7 \times 2)$ supercells [Figs. 9(b) and 9(c)], the ADB are separated by $5.25 \AA$ and $10.68 \AA$, respectively. The corresponding unit cell compositions of these defected structures are $\left(\mathrm{SiO}_{2}\right)_{16} 8 \mathrm{O}, \quad\left(\mathrm{SiO}_{2}\right)_{10} 5 \mathrm{O}$, and $\left(\mathrm{SiO}_{2}\right)_{14} 7 \mathrm{O}$. The sampling of the first Brillouin zone is done with a $(1 \times 4 \times 1),(2 \times 4 \times 1)$, and $(1 \times 4 \times 1) k$-point mesh, respectively.

The three ADB models have very similar formation energies, $\Delta E_{\text {form }}=-10.7 \mathrm{eV}$ for $\left(\mathrm{SiO}_{2}\right)_{16} 8 \mathrm{O}, \Delta E_{\text {form }}=-10.7 \mathrm{eV}$ for $\left(\mathrm{SiO}_{2}\right)_{10} 5 \mathrm{O}$, and $\Delta E_{\text {form }}=-10.6 \mathrm{eV}$ for $\left(\mathrm{SiO}_{2}\right)_{14} 7 \mathrm{O}$ model, respectively. They are only $0.1-0.2 \mathrm{eV}$ less stable than the $1 \mathrm{ML}$ model $A\left(E_{\text {form }}=-10.8 \mathrm{eV}\right)$. This strongly supports the proposed structure of ADB and provides more evidence for our atomic model of the $\mathrm{SiO}_{2} / \mathrm{Mo}(112)$ film. The small difference in formation energies between the simulated ADB models is confirmed by STM measurements, 
where the coexistence of all three models has been detected [Figs. 9(a)-9(c)].

\section{SUMMARY AND CONCLUSIONS}

We have determined the atomic structure of the thin crystalline $\mathrm{SiO}_{2}$ film on a $\mathrm{Mo}(112)$ substrate based on the excellent agreement between the results of DFT calculations and the new high-quality experimental data. The film consists of a two-dimensional network of corner-sharing $\mathrm{SiO}_{4}$ tetrahedra, with one oxygen atom from each tetrahedron bonding to Mo atoms of the protruding rows on the Mo(112) surface. The other three oxygen atoms form $\mathrm{Si}-\mathrm{O}-\mathrm{Si}$ bonds with the neighboring tetrahedra. There are three possible orientations of the $\mathrm{SiO}_{4}$ network with respect to the metal surface with bridge, top, and pseudo threefold hollow position of the interface $\mathrm{O}$ atoms with respect to Mo atoms of the protruding rows along the [11ㅣ] direction. Results of our calculations show that the structure with oxygen atoms in bridge positions is energetically the most favorable one. Simulations of the IRAS spectra, O $1 s$ core-level shifts, and STM images confirm this structure as the atomic model of the
$\mathrm{SiO}_{2} / \mathrm{Mo}(112)$ film. The topmost surface layer is fully saturated, which explains the essential inertness of the films towards water. The knowledge of the atomic structure of the silica film puts us into the position to study nucleation and growth of metals on flat, well-characterized silica surfaces at the atomic level. We note that a similar model of the cornersharing $\mathrm{SiO}_{4}$ network has been independently found by Giordano et al. ${ }^{17}$ However, their model corresponds to a less stable structure, where oxygen atoms occupy top sites with respect to Mo atoms of the protruding rows (1 ML model $B$, Fig. 5).

\section{ACKNOWLEDGMENTS}

The authors gratefully acknowledge financial support by the Deutsche Forschungsgemeinschaft (DFG) through the Sondersforschungsbereich SFB 546. T.K.T. and S.K. acknowledge the International Max Planck Research School "Complex Surfaces in Materials Science" for financial support and J.W. thanks the Alexander von Humboldt Stiftung. The calculations were carried out on the IBM p690 system of the Norddeutscher Verbund für Hoch- und Höchstleistungsrechnen (HLRN).
*Corresponding author: mas@chemie.hu-berlin.de

${ }^{1}$ D. W. Goodman, Surf. Rev. Lett. 2, 9 (1995).

${ }^{2}$ R. Franchy, Surf. Sci. Rep. 38, 195 (2000).

${ }^{3}$ H.-J. Freund, Angew. Chem., Int. Ed. 36, 452 (1997).

${ }^{4}$ G. Kresse, M. Schmid, E. Napetschnig, M. Shishkin, L. Köhler, and P. Varga, Science 308, 1440 (2005).

${ }^{5}$ F. Rohr, M. Bäumer, H.-J. Freund, J. A. Mejias, V. Staemmler, S. Müller, L. Hammer, and K. Heinz, Surf. Sci. Lett. 372, L291 (1997); F. Rohr, M. Bäumer, H.-J. Freund, J. A. Mejias, V. Staemmler, S. Müller, L. Hammer, and K. Heinz, ibid. 389, 391 (1997).

${ }^{6}$ T. Schroeder, M. Adelt, B. Richter, M. Naschitzki, M. Baümer, and H.-J. Freund, Surf. Rev. Lett. 7, 7 (2000).

${ }^{7}$ T. Schroeder, J. B. Giorgi, M. Bäumer, and H.-J. Freund, Phys. Rev. B 66, 165422 (2002).

${ }^{8}$ B. K. Min, A. K. Santra, and D. W. Goodman, Catal. Today 85, 113 (2003).

${ }^{9}$ M. S. Chen, A. K. Santra, and D. W. Goodman, Phys. Rev. B 69, 155404 (2004).

${ }^{10}$ B. K. Min, W. T. Wallace, A. K. Santra, and D. W. Goodman, J. Phys. Chem. B 108, 16339 (2004).

${ }^{11}$ B. K. Min, W. T. Wallace, and D. W. Goodman, J. Phys. Chem. B 108, 14609 (2004).

${ }^{12}$ S. Wendt, E. Ozensoy, T. Wei, M. Frerichs, Y. Cai, M. S. Chen, and D. W. Goodman, Phys. Rev. B 72, 115409 (2005).

${ }^{13}$ D. Ricci and G. Pacchioni, Phys. Rev. B 69, 161307(R) (2004).

${ }^{14}$ S. Wendt, M. Frerichs, T. Wei, M. S. Chen, V. Kempter, and D. W. Goodman, Surf. Sci. 565, 107 (2004).

${ }^{15}$ F. Liebau, Structural Chemistry of Silica (Springer-Verlag, Berlin,
1985).

${ }^{16}$ J. Weissenrieder, S. Kaya, J.-L. Lu, H.-J. Gao, S. Shaikhutdinov, H.-J. Freund, M. Sierka, T. K. Todorova, and J. Sauer, Phys. Rev. Lett. 95, 076103 (2005).

${ }^{17}$ L. Giordano, D. Ricci, G. Pacchioni, and P. Ugliengo, Surf. Sci. 584, 225 (2005).

${ }^{18}$ G. Kresse and J. Furthmüller, Comput. Mater. Sci. 6, 15 (1996).

${ }^{19}$ G. Kresse and J. Furthmüller, Phys. Rev. B 54, 11169 (1996).

${ }^{20}$ J. P. Perdew, J. A. Chevary, S. H. Vosko, K. A. Jackson, M. R. Pederson, D. J. Singh, and C. Fiolhais, Phys. Rev. B 46, 6671 (1992).

${ }^{21}$ P. E. Blöchl, Phys. Rev. B 50, 17953 (1994).

${ }^{22}$ G. Kresse and D. Joubert, Phys. Rev. B 59, 1758 (1999).

${ }^{23}$ H. J. Monkhorst and J. D. Pack, Phys. Rev. B 13, 5188 (1976).

${ }^{24}$ J. Tersoff and D. R. Hamann, Phys. Rev. B 31, 805 (1985).

${ }^{25}$ A. P. Scott and L. Radom, J. Phys. Chem. 100, 16502 (1996).

${ }^{26}$ R. K. Sato and P. F. McMillan, J. Phys. Chem. 91, 3494 (1987).

${ }^{27}$ J. F. Scott and S. P. S. Porto, Phys. Rev. 161, 903 (1967).

${ }^{28}$ S. Baroni, S. de Gironcoli, A. Dal Corso, and P. Giannozzi, Rev. Mod. Phys. 73, 515 (2001).

${ }^{29}$ K. Reuter and M. Scheffler, Phys. Rev. B 65, 035406 (2002).

${ }^{30}$ J. F. Moulder, W. F. Stickle, P. E. Sobol, and K. D. Bomben, Handbook of X-ray Photoelectron Spectroscopy (Perkin-Elmer Corporation, Eden Prairie, MN, 1992).

${ }^{31}$ T. Schroeder, J. Zegenhagen, N. Magg, B. Immaraporn, and H.-J. Freund, Surf. Sci. 552, 85 (2004).

${ }^{32}$ C. T. Kirk, Phys. Rev. B 38, 1255 (1988).

${ }^{33}$ I. N. Yakovkin, Surf. Rev. Lett. 12, 449 (2005). 